\title{
Canarypox virus expressing infectious bursal disease VP2 protein as immunogen for chickens
}

\author{
Flavia Adriana Zanetti ${ }^{1}$, María Daniela Conte Grand ${ }^{1}$, Romina Cristina Mitarotonda ${ }^{2}$, \\ Oscar Alberto Taboga ${ }^{1,2}$, Gabriela Calamante ${ }^{2}$ \\ ${ }^{1}$ Consejo Nacional de Investigaciones Científicas y Tecnológicas, Ciudad Autónoma de Buenos Aires, \\ Argentina. \\ ${ }^{2}$ Instituto de Biotecnología, Centro de Investigaciones en Ciencias Veterinarias y Agronómicas, \\ Instituto Nacional de Tecnología Agropecuaria, Castelar, Buenos Aires, Argentina.
}

Submitted: September 12, 2012; Approved: September 9, 2013.

\begin{abstract}
Canarypox viruses (CNPV) carrying the coding sequence of VP2 protein from infectious bursal disease virus (IBDV) were obtained. These viruses were able to express VP2 protein in vitro and to induce IBDV-neutralizing antibodies when inoculated in specific pathogen-free chickens demonstrating that CNPV platform is usefulness to develop immunogens for chickens.
\end{abstract}

Key words: canarypox virus, viral vector, IBDV, VP2 protein.

Canarypox virus (CNPV), a member of the avipoxvirus genus, has been successfully evaluated as recombinant expression vectors for vaccines development. In this sense, the CNPV platform has been used to give rise protective immune response against various pathogens in different mammalian species (reviewed in Poulet et al., 2007; Brun et al., 2008). Moreover, some of them have reached the market as licensed products commercialized by Merial (a Sanofi Pasteur Company).

Based on the fact that no reports have been published using this viral vector to prevent diseases in chickens, we decided to obtain and evaluate recombinant CNPV as immunogen for that species selecting VP2 protein from infectious bursal disease virus (IBDV) as a model antigen. IBDV is the etiologic agent of infectious bursal disease (IBD), a highly contagious and severe disease that causes significant economic losses in the poultry industry worldwide. IBDV replicates primarily in B lymphocyte precursors developing within the bursa of Fabricious (Müller et al., 2003). Depending on the virulence of IBDV strain and the age of the infected chickens, IBD is manifested in two ways. In some cases occur with clinical symptoms and may cause up to $20 \%$ or more mortality in chickens 3 weeks of age and older. Otherwise, and more important, is evidenced by a severe, prolonged immunosuppression of chickens infected at an early age (Eterradossi and Saif, 2008). The VP2 mature protein (of 441 residues) is the only structural component of the IBDV icosahedral capsid, and thus responsible to the interaction with host cell receptors. VP2 constitutes the major host-protective antigen as it contains conformation-dependent epitopes responsible for eliciting neutralizing antibodies (Müller et al., 2003).

In this work, we obtained recombinant canarypox viruses that express IBDV VP2 protein and showed their immunogenicity when inoculated in specific pathogen-free (SPF) chickens.

The first step was the construction of the transference vector (named TV-CN048-VP2) carrying foreign genes flanked by viral regions (position 57,247, inside CNPV046, to 59,528, inside CNPV048, (Tulman et al., 2004)) which serve as points of recombination with CNPV genome. The selected gene was the coding sequence for VP2 mature protein from the Argentinean IBDV isolate LD-847-04, serotype I (vp2 gene, NCBI accession No. JF965438). This nucleotide sequence was obtained as described before (Zanetti et al., 2012a) and subcloned into TV-CN048-GUS plasmid which was previously designed and built in our laboratory (Zanetti et al., 2012b). The final construction was the transference vector $(8,452 \mathrm{bp}$, Figure 1A), which carried $v p 2$ gene downstream of the poxviral synthetic

Send correspondence to G. Calamante. Instituto de Biotecnología, Centro de Investigaciones en Ciencias Veterinarias y Agronómicas, Instituto Nacional de Tecnología Agropecuaria, P.O. Box 25 (B1712WAA) Castelar, Buenos Aires, Argentina. E-mail: calamante.gabriela@inta.gob.ar. 
early/late promoter $(\mathrm{pE} / \mathrm{L})$ and the expression cassette for the GUS enzyme (uid $A$ gene under regulation of vaccinia virus $\mathrm{H} 6$ gene promoter), both flanked by CNPV genomic sequences.

The $v p 2$ gene identity was confirmed by DNA sequencing using an ABI PRISM 3130 genetic analyzer (Applied Biosystem, Japan). Then, the final plasmid TVCN048-VP2 was transfected, by Lipofectin ${ }^{\mathrm{TM}}$ (Invitrogen, Carlsbad, CA, USA), into SPF primary chicken embryo fibroblasts (CEFs) previously infected with CNPV (Abbatista95 vaccine strain provided by LaDiPreVet laboratory, La Plata, Argentina). Recombinant viral clones forming GUS positive plaques were visualized with X-Gluc (5-bromo-4-chloro-3-indolyl- $\beta$-D-glucuronic acid, Inalco, Italy) overlay. After five rounds of purification, $100 \%$ of blue plaques were visualized and CN048-VP2 viruses were amplified and molecularly characterized. Firstly, total DNA

A

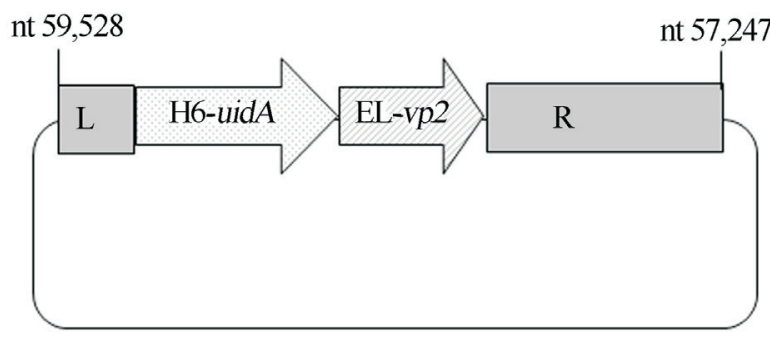

C

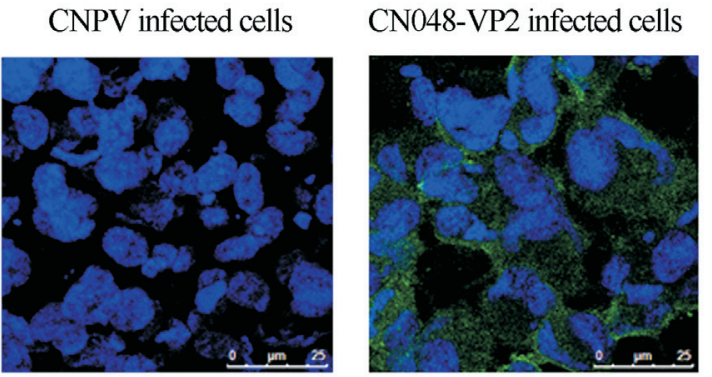

$\mathrm{D}$

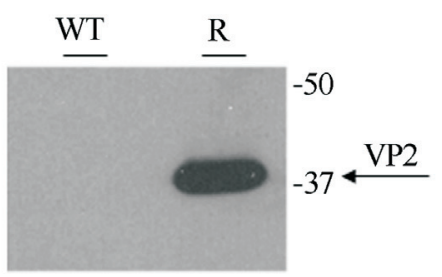

extracted from infected CEFs was analysed by Southern Blot (Ferrer et al., 2011). The purity of recombinant viruses (absence of wild type genome) and the insertion of the VP2 expression cassette were confirmed by hybridization with ${ }^{32} \mathrm{P}$-labelled CNPV048 probe (genomic positions 58,948 to $59,528)$ or $v p 2$ gene probe, respectively (Figure 1B). Thereafter, the expression of VP2 protein from recombinant viruses was evaluated by Indirect Immunofluorescence (IIF) and Western blot (WB) assays in DF1 (ATCC, CRL12203) infected cells as previously described (Zanetti et al., 2012a). The expression of VP2 protein (green fluorescence) was clearly observed in the cytoplasm of CN048VP2 infected cells but no fluorescence was evidenced in CNPV-infected cells (Figure $1 \mathrm{C}$ ). Besides, a band of approximately $37 \mathrm{kDa}$ was only detected by WB in DF1 cells infected with the recombinant CN048-VP2 (Figure 1D). Taken together, these results demonstrated the capability of

B

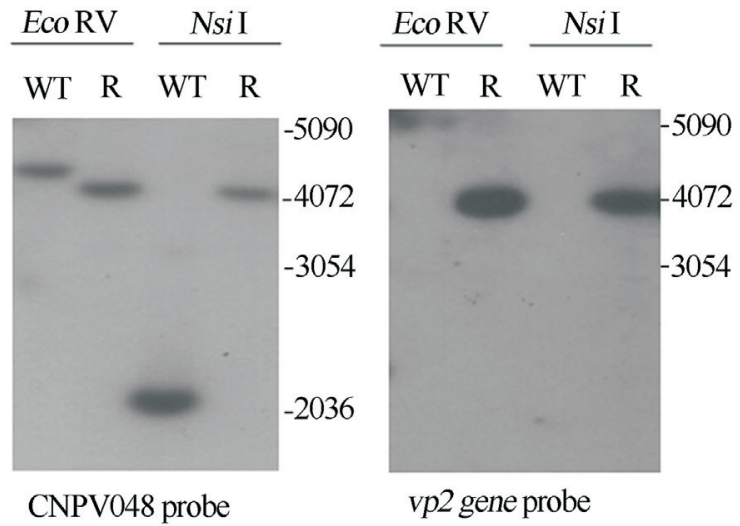

E

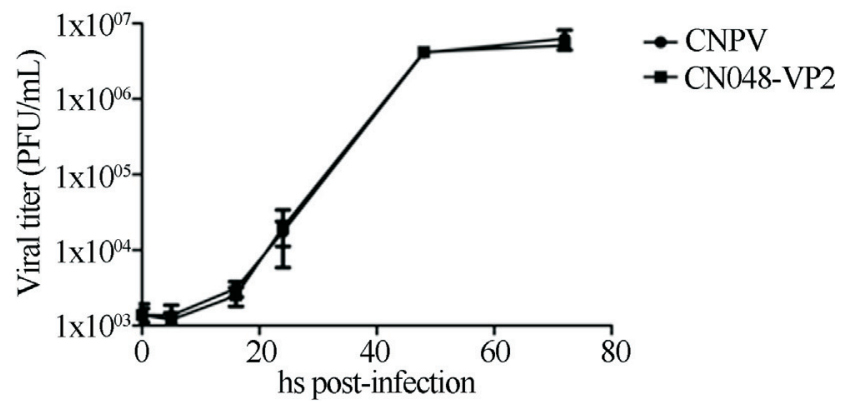

Figure 1 - Molecular characterization of CN048-VP2 recombinant virus. (A) Scheme of the transference vector TV-CN048-VP2. L: 600 bp fragment started in position 59,528. R: 1,700 bp fragment until position 57,247 encompassing the final region of CNPV048 gene, the complete CNPV047 gene and the initial region of CNPV046 gene. (B) Autorradiographies of Southern blot analysis using a $600 \mathrm{bp}$ fragment of CNPV048 gene (positions 58,927 to 59,528 ) or vp 2 gene as ${ }^{32} \mathrm{P}$-labelled probes. (C) Indirect immunofluorescence assay in DF1 cells infected with CN048-VP2 or non-recombinant CNPV viruses; white bars represents $25 \mu \mathrm{m}$. (D) Western blot assay: protein extracts were resolved in 12\% SDS-PAGE and VP2 protein (indicated by an arrow) was detected using a rabbit polyclonal serum against IBDV-VP2 protein. Apparent molecular masses are indicated in kDa. WT: non-recombinant CNPV virus, R: recombinant CN048-VP2 virus. (E) Multiple-step growth curve of CNPV and CN048-VP2 in CEFs. The PFU/mL at each point is the mean of two independent experiments; errors bars represent standard deviations. 
CN048-VP2 virus to in vitro express the IBDV VP2 protein with the predicted molecular weight for the mature protein. Thereafter, replication ability of recombinant and parental CNPVs was assessed by performing a multiple-step growth curve. Briefly, CEFs were infected at 0.01 plaque-forming units (PFU)/cell with CNPV or CN048-VP2 viruses. Supernatant and cells were collected together at different hours post infection $(0,5,16,24,48$ and $72 \mathrm{~h})$ and frozen/thawed three times before tittering by duplicate in CEFs. The viral titer $(\mathrm{PFU} / \mathrm{mL})$ was calculated as the average value of the viral titers obtained in two independent experiments. Recombinant and wt CNPVs had the same growth curves indicating that the insertion of foreign sequences interrupting CNPV048 gene did not modify the viral replication capability in CEFs (Figure 1E). Then, the genetic stability of the recombinant viruses was evaluated by ten consecutive multiple-step growth passages on CEFs. After the $10^{\text {th }}$ passage recombinant viruses produced $100 \%$ of blue plaques and the expression of VP2 protein was confirmed by WB (data not shown).

Then, the immunogenicity of CN048-VP2 was evaluated in SPF White Leghorn chickens randomly separated in four groups of five birds each. The chickens (11-days-old) were intramuscularly (i.m.) immunized with CNPV or CN048-VP2 $\left(5.5 \times 10^{5} \mathrm{PFU} / \mathrm{bird}\right)$ or orally vaccinated with D78 live vaccine $\left(2.7 \times 10^{3} \mathrm{PFU} / \mathrm{bird}\right)$ or PBS $(50 \mu \mathrm{L})$. All birds were boosted at 32 and 53 days-old with the same inocula. At different times (11, 32, 53 and 74 days old) blood samples were collected from the wing vein to evaluate the induced specific humoral response. The experiment was performed in the animal facilities at the Biotechnology Institute (INTA) in compliance with guidelines of Institutional Committee for the Use and Care of Experimentation Animals (CICUAE-INTA) that approved the protocol 18/2012 based on both National Institute of Health Guide for the care and use of laboratory animal and Directive 2010/63/EU of the European Parliament on the protection of animals used for scientific purposes. Sera from the same experimental group were pooled and analyzed by an in vitro IBDV neutralization test as described before (Jackwood et al., 1982; Zanetti et al., 2012a). As expected, neither the sera obtained from 11 days old birds (pre-immune status) nor the samples collected from CNPV or PBS groups showed IBDV-specific antibodies. However, IBDV neutralizing antibodies were detected in sera from chickens vaccinated with CN048-VP2 or D78. Besides, the seroneutralizing titer increased after each CN048-VP2 immnunization indicating that the anti-vector immunity did not affect the efficacy of the booster vaccinations. The IBDVD78 live vaccine induced higher level of neutralizing antibodies than the recombinant virus (Table 1).

At present, in the poultry industry, IBD is controlled by immunization with attenuated live or inactivated IBDV vaccines. However, these vaccines have several disadvantages. The inactivated vaccines are expensive and must be
Table 1 - IBDV-neutralizing titers in the sera of vaccinated birds

\begin{tabular}{lccc}
\hline & \multicolumn{3}{c}{ Age (days) } \\
\cline { 2 - 4 } & 32 & 53 & 74 \\
\hline CN048-VP2 & 16 & 32 & 512 \\
IBDV-D78 & 512 & 1024 & 1024 \\
\hline
\end{tabular}

Seroneutralizing titers are expressed as the reciprocal of the last serum dilution that completely neutralized 100 TCID50 (50\% tissue culture infective doses) units of IBDV strain LZD. CN048-VP2: recombinant CNPV virus expressing IBDV VP2 mature protein, IBDV-D78: live commercial IBDV vaccine (strain D78). IBDV-specific antibodies were negative both in the sera from 11 days old chicks (pre-immune) as in the samples collected from CNPV or PBS immunized birds.

administered to birds previously immunizated with live vaccines (Müller et al., 2003). Attenuated IBDV vaccines have to be applied once the IBDV maternal antibodies have decreased and could cause moderate bursal atrophy and/or immunosuppresion. This fact allowed both the infection with other pathogens and poor immune response to other vaccines. More recently, the use of an immune complex vaccine (containing a live IBDV strain and an anti-IBDV hyperimmune serum, [Müller et al., 2003]) or a turkey herpesvirus-based vector vaccine (recombinant HVT expressing IBDV-VP2 protein, [Poulet et al., 2007]) has been implemented to the control of IBD in commercial poultry populations. Although these vaccines are effective in the presence of maternal antibodies inducing lifelong immunity, they have the major disadvantage of their high cost due to the addition of anti-serum (immune complex) or to the requirements of a cold chain to maintain the viability of HVT vector. In contrast, the high physical stability of CNPV-based viral vector allows the formulation of either freeze-dried or liquid vaccines with shelf-lives up to 2 years at room temperture (Poulet et al., 2007).

In this report, we demonstrated that CN048-VP2 viruses were capable to elicit an IBDV specific humoral response with the presence of neutralizing antibodies in the vaccinated chickens. Due to neutralizing epitopes located on VP2 are discontinuous, it could be assumed that the VP2 protein expressed from CN048-VP2 had a correct conformational structure. In addition to that, we demonstrated that anti-canarypox vector immune response would not interfere with the following immunizations since the values of IBDV-seroneutralizing titers increased after each booster. This result agreed with observations of Poulet et al. (2007) who described that canarypox viral vectors are chosen for vaccination programs that require several boosters to achieve protective immunity. The immunization of chickens with CN048-VP2 viral vector elicited lower neutralizing antibody titers than replicative D78 live vaccine, but the poxvirus vector could also be inducing a cellular immune response contributing to protection against IBDV infections (Müller et al., 2003). For that reason, further experiments have to be performed to evaluate both protection and cellular immune response induced by CN048-VP2 
vaccination of chickens (with and without IBDV maternal antibodies). Besides, other routes of administration of CN048-VP2 consistent with the use in poultry industry such as subcutaneous immunization or wing-web scarification will be evaluated. Finally, a recombinant CNPV co-expressing cytokines with VP2 protein will be obtained with the aim to improve the specific immune response in chickens.

In conclusion, we demonstrated that recombinant canarypox viruses could be effective for developing novel vaccines against poultry diseases. To our knowledge this is the first report of immunization of chickens with canarypox virus-based vector.

\section{Acknowledgments}

This work was supported by grants AERG 232141 from Instituto Nacional de Tecnología Agropecuaria (INTA) and PICT2004-25309 and PICT2008-0400 from Agencia Nacional de Promoción Científica y Tecnológica (ANPCyT), Argentina. FAZ was supported by an external fellowship for junior researchers from CONICET, Argentina. The authors are very grateful to Dr. S. Samus for kindly providing the attenuated strain Abbatista95 of canarypox virus and to Dr. O. Zabal, Mrs. M. J. Monaco and Mr. S. Díaz for preparing the primary chicken embryo fibroblasts, technical assistance and care of the chickens, respectively. We also thank Dr. J.F. Rodríguez from Centro Nacional de Biotecnología (CNB), Madrid, Spain for assistance in immunofluorescence assay and for providing antiVP2 serum.

\section{References}

Brun A, Albina E, Barret T, Chapman DAG, Czub M, Dixon LK, Keil G M, Klonjkowski B, Le Potier MF, Libeau G, Ortego
J, Richardson J, Takamatsu H H (2008) Antigen delivery systems for veterinary vaccine development viral-vector based delivery systems. Vaccine 26:6508-6528.

Eterradossi N, Saif Y M (2008) Infectious bursal disease. In: Saif, Y.M. (ed) Diseases of Poultry. 12th Ed. Blackwell Publishing, Ames, pp 185-208.

Ferrer MF, Del Médico Zajac MP, Zanetti FA, Valera AR, Zabal O, Calamante G (2011) Recombinant MVA expressing secreted glycoprotein D of BoHV-1 induces systemic and mucosal immunity in animal models. Viral Immunol 24:331-339.

Jackwood DJ, Saif YM, Moorhead PD, Dearth R N (1982) Infectious bursal disease virus and Alcaligenes faecalis infections in turkey. Avian Dis 26:365-374.

Müller H, Islam MR, Raue R (2003) Research on infectiuos disease-the past, the present and the future. Vet Microbiol 97:153-165.

Poulet H, Minke J, Pardo MC, Juillard V, Nordgren B, Audonnet JC (2007) Development and registration of recombinant veterinary vaccines. The example of the canarypox vector platform. Vaccine 25:5606-5612.

Tulman ER, Afonso CL, Lu Z, Zsak L, Kutish GF, Rock DL (2004) The genome of canarypox virus. J Virol 78:353-366.

Zanetti FA, Del Médico Zajac MP, Taboga AO, Calamante G (2012a) Evaluation of modified vaccinia virus Ankara expressing VP2 protein of infectious bursal disease virus as immunogen in chickens. J Vet Sci 13:199-201.

Zanetti F, Rudak L, Micucci M, Conte Grand D, Luque A, Russo S, Taboga O, Pérez O, Calamante G (2012b) Development and preliminary assessment of a recombinant canarypox virus as an antirabic vaccine candidate. Rev Argent Microbiol 44:75-84.

All the content of the journal, except where otherwise noted, is licensed under a Creative Commons License CC BY-NC. 